\title{
Nagashima-type palmoplantar keratosis in a Chinese Han population
}

\author{
JIA ZHANG $^{1 *}$, GUOLONG ZHANG ${ }^{2 *}$, CHENG NI $^{1}$, RUHONG CHENG $^{1}$, \\ JIANYING LIANG ${ }^{1}$, MING $\mathrm{LI}^{1}$ and ZHIRONG YAO ${ }^{1}$ \\ ${ }^{1}$ Department of Dermatology, Xinhua Hospital Affiliated to Shanghai Jiaotong University School of Medicine, \\ Shanghai 200092; ${ }^{2}$ Department of Phototherapy, Shanghai Skin Disease Hospital, Shanghai 200050, P.R. China
}

Received July 11, 2015; Accepted May 13, 2016

DOI: $10.3892 / \mathrm{mmr} .2016 .5757$

\begin{abstract}
Nagashima-type palmoplantarkeratosis (NPPK) is an autosomal recessive form of palmoplantar keratoderma (PPK), which is caused by mutations in the SERPINB7 gene. NPPK has only been reported in Japanese and Chinese populations. The present study was conducted on 12 unrelated Chinese patients who were clinically predicted to suffer from NPPK. Mutation screening was performed by direct sequencing of the entire coding regions of SERPINB7, SLURP1, AQP5, CSTA, KRT1 and KRT9 genes. Direct sequencing of SERPINB7 revealed five homozygous founder mutations (c.796C $>$ T) and four compound heterozygous mutations in nine patients, including one novel mutation (c.122_127delTGGTCC). Nine out of the 12 patients were diagnosed with NPPK due to SERPINB7 pathogenic mutations, and the results expanded the known mutation spectrum of NPPK. Taking the other seven reported Chinese patients, who had been definitively diagnosed with NPPK by genetic testing, into account, the present study further demonstrated that NPPK is a common entity in Mainland China, and c.796C>T is the most prevalent mutation and exerts a founder effect. Furthermore, the NPPK cases described in the current study presented a consistently mild phenotype, as compared with the degrees of phenotypic variability associated with other types of relatively severe PPK, including Mal de Meleda and Olmsted syndrome.
\end{abstract}

Correspondence to: Professor Zhirong Yao or Dr Ming Li, Department of Dermatology, Xinhua Hospital Affiliated to Shanghai Jiaotong University School of Medicine, 1665 Kongjiang Road, Shanghai 200092, P.R. China

E-mail: zryaosmu@sohu.com

E-mail: aypyslm@163.com

*Contributed equally

Key words: Nagashima-type palmoplantar keratosis, SERPINB7, Chinese

\section{Introduction}

Nagashima-type palmoplantar keratosis (NPPK; OMIM \#615598) is an autosomal recessive form of palmoplantar keratoderma (PPK), which exhibits a relatively high incidence and has only been reported in Japanese and Chinese populations $(1,2)$. The characteristic features of NPPK are erythema and hyperkeratosis of the palms and soles, with sharp demarcation that mainly extends to the wrists, ankles, Achilles tendon area, and the dorsal aspects of the fingers and toes (1). Furthermore, other frictional regions, such as knees and elbows, may be involved. Some patients with NPPK also exhibit associated palmoplantar features, including a white, spongy appearance within 10 min of water exposure, hyperhidrosis and fungal infections (3).

The clinical features of diffuse PPK and the recessive mode of inheritance are not unique characteristics for NPPK, but are also associated with autosomal recessive exfoliative ichthyosis (AREI; OMIM \#607936), which is caused by mutations in cystatin A (CSTA) (4), and an atypical mild form of Mal de Meleda (MDM; OMIM \#248300), which is caused by mutations in secreted LY6/PLAUR domain containing 1 (SLURP1) (5). Other types of autosomal dominant diffuse PPK with de novo mutations include Unna-Thost type PPK [OMIM \#600962; corresponding gene, keratin (KRT)1], Vorner type PPK (OMIM \#144200; corresponding gene, KRT9/KRT1) and Bothnian type PPK [OMIM 600231; corresponding gene, aquaporin 5 (AQP5)], which must be differentiated from NPPK (3).

Mutations in the serpin peptidase inhibitor, clade B (ovalbumin), member 70 (SERPINB7) gene, which encodes a member of the serine protease inhibitor superfamily, results in a complete loss of protease inhibitory activity. SERPINB7 mutations were reported to be responsible for NPPK in 2013 (3).

At present, only 31 unrelated, molecularly diagnosed cases of NPPK associated with seven distinct pathogenic SERPINB7 mutations in the homozygous or compound heterozygous state have been reported in the literature. These cases include the most popular founder mutation c.796C $>\mathrm{T}$, and other potentially frequent mutations c.218_219del2ins 12 , c. $336+2 \mathrm{~T}>\mathrm{G}$, c.455-1G $>$ A, c.455G $>$ T, c.522dupT and c.650_653delCTGT $(2,3,6,7)$. Among these studies, only one report regarding 
seven cases of NPPK associated with four different SERPINB7 mutations was available in Mainland China (2).

The present study investigated 12 suspected Chinese patients with NPPK. Nine of the participants were definitely diagnosed with NPPK by molecular analysis.

\section{Materials and methods}

Subjects. The present study was approved by the Institutional Review Board of Xinhua Hospital, Shanghai Jiaotong University School of Medicine (Shanghai, China). All participants provided written informed consent. Patients suspected as having NPPK were recruited by experienced dermatologists from Xinhua Hospital and Shanghai Skin Hospital (Shanghai, China). In total, 12 probands (8 females and 4 males), nearly all of their parents (except for the parents of Patients 11 and 12 , and the father of Patient 10), and 100 population-matched healthy controls were enrolled in the present study between August 2006 and December 2014. All probands were genetically unrelated ethnic Han Chinese, which exhibited non-progressive, symmetrical, diffuse erythema and hyperkeratosis over the palms and soles from infancy. Elbow and knee involvement was not observed. The clinical appearance of three patients (Patients 1, 3 and 10; 8 months old, 19 years old and 22 years old, respectively) is presented in Figs. 1-3. Clinical details were available for 10 of the 12 patients. The age group ranged between 8 months and 51 years. The age of onset was $\sim 3$ months (Table I). No family history and consanguinity was identified in this cohort.

Methods. Peripheral blood samples were collected from all participants for DNA extraction. DNA was extracted using a TIANamp Blood DNA kit (Tiangen Biotech Co., Ltd., Beijing, China) and PCR primers were designed flanking all coding exons and intron-exon boundaries of six genes (SERPINB7, SLURP1, AQP5, CSTA, KRT1 and KRT9) using Primer Premier 5.0 software (Premier Biosoft, Palo Alto, CA, USA; Table II). Genomic DNA samples were amplified by standard polymerase chain reaction and the PCR protocol was as follows: i) Denaturation, $94^{\circ} \mathrm{C}$ for $5 \mathrm{~min}$; ii) 31 cycles of denaturation at $94^{\circ} \mathrm{C}$ for $30 \mathrm{sec}$, annealing for $30 \mathrm{sec}$ at temperatures according to the primers for each fragment, and extension at $72^{\circ} \mathrm{C}$ for $1 \mathrm{~min}$; iii) extension, $72^{\circ} \mathrm{C}$ for $1 \mathrm{~min}$; and iv) extension, $4^{\circ} \mathrm{C}$ for $5 \mathrm{~min}$. PCR was repeated 10-20 times. Sanger sequencing was performed using an ABI PRISM ${ }^{\circledR} 3730$ automated sequencer (Applied Biosystems; Thermo Fisher Scientific, Inc., Waltham, MA, USA). The SERPINB7 gene, which is associated with autosomal recessive NPPK, was tested initially. If no corresponding mutations in two alleles were identified, the other five genes were sequentially sequenced. Identified mutations were respectively confirmed in the unaffected family members (if available) and 100 population-matched healthy controls (if mutation is novel).

\section{Results}

Mutations identified in SERPINB7 (GenBank accession number: NM_001040147.2) are summarized in Table I. The present study detected five homozygous founder mutations (c.796C $>\mathrm{T}$ ) and four compound heterozygous mutations (c.796C $>\mathrm{T}$ combined with c.455G $>\mathrm{T}$, c.522dupT or c.122_127delTGGTCC) in nine patients with NPPK (Fig. 4). Among the mutations, the in-frame deletion mutation c.122_127delTGGTCC has not previously been reported, and was also absent in the 100 population-matched healthy controls. Furthermore, indexed unaffected parents in the present cohort were all heterozygous carriers. Notably, the other three patients were revealed to harbor one heterozygous founder mutation (c.796C>T) in SERPINB7, whereas no pathogenic mutations were detected in the five remaining candidate genes.

\section{Discussion}

All of the seven previously reported pathogenic mutations associated with NPPK (c.218_219del2ins12, c.336+2T $>$ G, c.455-1G>A, c.455G>T, c.522dupT, c.650_653delCTGT, c.796C $>$ T) are nonsense/frameshift/splice site mutations, which form premature stop codons and truncate the protein, thus suggesting that missense variants without splicing effects tend to be non-pathogenic polymorphisms. The novel mutation c.122_127delTGGTCC (p.Leu41_Val42del) identified in the present cohort was estimated to cause an in-frame deletion of two amino acid residues (leucine and valine). Considering patient 10 exhibited the typical features of NPPK (Fig. 3), and the in-frame deletion mutation was not detected in 200 alleles from the 100 healthy controls, it was predicted that this mutation may shorten the protein, and exert pathogenic effects resulting in an NPPK phenotype.

Notably, three suspected patients with typical palmoplantar lesions of NPPK were all shown to harbor one heterozygous founder mutation (c.796C>T) in SERPINB7, whereas no pathogenic mutations were identified in the remaining five candidate genes responsible for analogous genodermatoses. It has previously been reported that female carriers with missense SLURPI mutations may exhibit mild palmar lesions (8), whereas a heterozygous SERPINB7 mutation did not induce any palmoplantar abnormalities, thus indicating that either of the alleles can retain protein activity of SERPINB7 and sustain normal skin structure. Therefore, the three suspected cases very probably suffer from NPPK. In addition, novel mutations in the other allele may be undetectable large deletions, or mutations located in other loci of SERPINB7, including deep introns and 5'/3'-untranslated regions, which require more intensive investigations, such as RNA testing or next-generation sequencing. Unfortunately, these samples are not available for retesting.

The major etiological factor associated with MDM is consanguineous marriage $(9,10)$, and MDM has been diagnosed in the Mediterranean region, the Middle East, Europe and East Asia. Conversely, NPPK appears to be an Asian-only endemic (11), the prevalence rate of which has been estimated to be 1.2/10,000 and 3.1/10,000 in Japanese and Chinese Han populations, respectively (3). Furthermore, the most common mutation (c.796C > T) exerts a founder effect and contributes to NPPK etiology (2).

In order to detect other SERPINB7 variants with a worldwide range that harbor the potential for being pathogenic, they were searched for using the 1000 Genomes Browser Version 3.0.2 (http://www.ncbi.nlm.nih.gov/variation/tools/1000genomes/). 


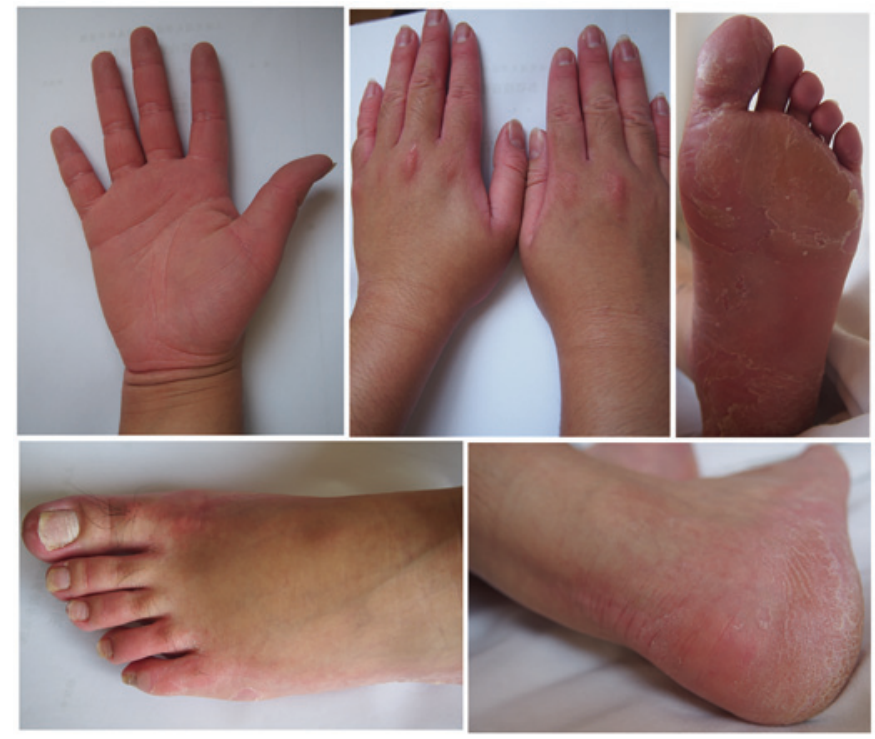

Figure 1. Clinical appearance of a 19-year-old patient with a homozygous c.796C $>$ T mutation.

The corresponding results are summarized in Table III. Furthermore, in combination with previous studies, the c.218_219del2ins12 mutation has only been detected in the Japanese population; the c.522dupT and c.650_653delCTGT mutations were only detected in the Chinese Han population; and four known pathogenic mutations (c.336+2T $>\mathrm{G}$, c. $455-1 \mathrm{G}>\mathrm{A}, \mathrm{c} .455 \mathrm{G}>\mathrm{T}$ and c.796C $>\mathrm{T}$ ) existed in both populations $(2,3,7)$. Notably, c.455G $>\mathrm{T}$ and c.522dupT have been reported in two unrelated patients, and the c. $455 \mathrm{G}>\mathrm{T}$ mutation has a high allele frequency of 0.0025 , in the Chinese Han population, thus suggesting a potential founder effect (7).

SERPINB7 is expressed in the epidermis of the whole body, and belongs to a cluster of clade-B serpins that inhibit serine proteases and protect cells from exogenous and endogenous proteolysis (12). Notably, absence of the critical reactive site loop (P17-P50; amino acid residues, 331-352) is predicted to be responsible for skin abnormalities in NPPK. All of the identified mutations, with the exception of the novel mutation detected in the present cohort, are expected to truncate the reactive site loop, thus resulting in NPPK phenotypes. The precise function of SERPINB7 remains poorly defined. Sakabe et al (13) speculated that the pathogenesis of NPPK may be associated with the effects of $\mathrm{T}$ cells infiltrating into the skin, thus suggesting a potential treatment with topical drugs that inhibit $\mathrm{T}$ cell infiltration, such as tacrolimus ointment.

The causal genes of PPK are associated with variable phenotypes (5). SLURPI mutations may cause mild MDM without plantar, nail, knee and elbow involvement, as well as severe complications, including higher occurrence of malignant melanoma in the hyperkeratotic area (14). TRPV3 mutations result in Olmsted syndrome (OMIM \#614594) with bilateral mutilating palmoplantar hyperkeratosis and periorificial keratotic plaques, along with focal PPK with mild acropodium deformation (15). SERPINB7-associated NPPK has been reported to exhibit little phenotypic heterogeneity, corresponding hyperkeratotic lesions with a reddish

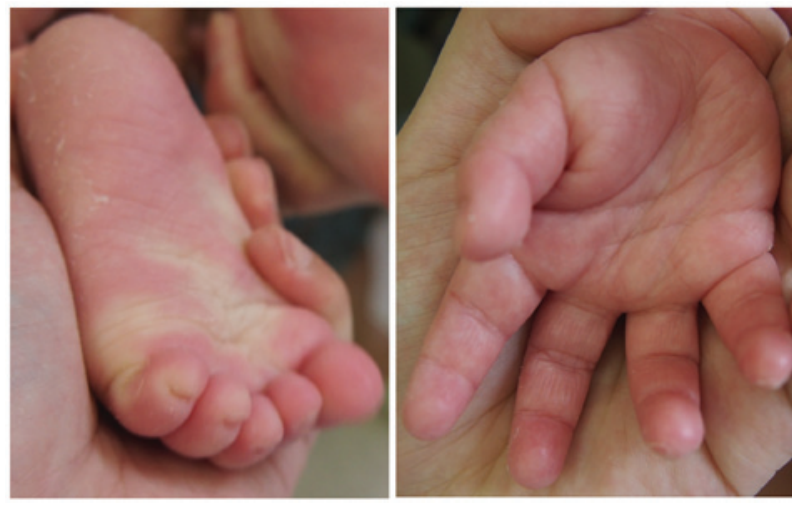

Figure 2. Clinical appearance of an 8-month-old patient with a homozygous c.796C $>$ T mutation.

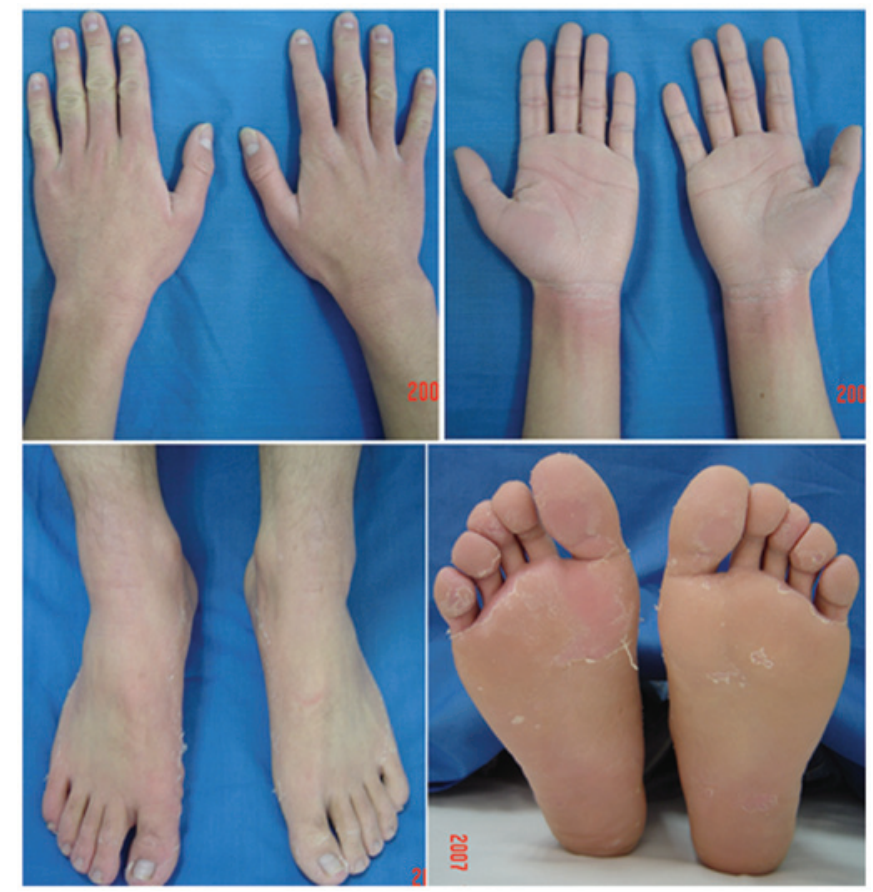

Figure 3. Clinical appearance of a 22 -year-old patient with a novel c.122_127delTGGTCC mutation.

appearance that predominantly occur in chronic mechanical stress-exposed areas of the skin, and a non-progressive disease course (3), all of which were also observed in the present cohort (Figs. 1-3). These clinical phenomena suggested that except for the distinct pathogenic mechanisms of corresponding genes, external stimuli, such as injury and friction, may also be crucial factors associated with the occurrence of NPPK phenotypes, which is similar to the findings of our previous report regarding the progression of MDM (16). Furthermore, taking into account other potential factors, including epigenetic alterations, modifier genes and ethnic background, sometimes diseases with a diffuse PPK appearance are analogous and hard to differentiate. For instance, the clinical photographs in previously published studies regarding clinically suspected NPPK seem to bear close similarity to mild or early stage MDM due to the yellowish palmoplantar lesions $(5,17)$, which differ from the usual 
Table I. Identified SERPINB7 mutations in the present cohort.

\begin{tabular}{|c|c|c|c|c|c|c|}
\hline \multirow[b]{3}{*}{$\begin{array}{l}\text { Patient } \\
\text { no. }\end{array}$} & \multirow[b]{3}{*}{$\begin{array}{l}\text { Gender/ } \\
\text { age }\end{array}$} & \multirow[b]{3}{*}{$\begin{array}{l}\text { Age at first } \\
\text { symptom }\end{array}$} & \multicolumn{4}{|c|}{ Molecular results } \\
\hline & & & \multicolumn{2}{|c|}{ Allele 1} & \multicolumn{2}{|c|}{ Allele 2} \\
\hline & & & $\begin{array}{c}\text { Nucleotide } \\
\text { change }\end{array}$ & $\begin{array}{l}\text { Amino acid } \\
\text { change }\end{array}$ & $\begin{array}{l}\text { Nucleotide } \\
\text { change }\end{array}$ & $\begin{array}{l}\text { Amino acid } \\
\text { change }\end{array}$ \\
\hline 1 & $\mathrm{M} / 8 \mathrm{~m}$ & $2 \mathrm{~m}$ & c. $796 \mathrm{C}>\mathrm{T}$ & p.R266* & c. $796 \mathrm{C}>\mathrm{T}$ & p.R266* \\
\hline 2 & $\mathrm{~F} / 2 \mathrm{y}$ & $1 \mathrm{w}$ & c. $796 \mathrm{C}>\mathrm{T}$ & p.R266* & c.522dupT & p.Val175fs \\
\hline 3 & $\mathrm{~F} / 19 \mathrm{y}$ & $3 \mathrm{~m}$ & c. $796 \mathrm{C}>\mathrm{T}$ & p.R266* & c.796C >T & p.R266* \\
\hline 4 & $\mathrm{M} / 4 \mathrm{y}$ & $6 \mathrm{~m}$ & c. $796 \mathrm{C}>\mathrm{T}$ & p.R266 & c. $796 \mathrm{C}>\mathrm{T}$ & p.R266 \\
\hline 5 & $\mathrm{M} / 16 \mathrm{y}$ & $3 \mathrm{~m}$ & c. $796 \mathrm{C}>\mathrm{T}$ & p.R266* & $?$ & $?$ \\
\hline 6 & $\mathrm{~F} / 26 \mathrm{y}$ & $2 \mathrm{~m}$ & c. $796 \mathrm{C}>\mathrm{T}$ & p.R266* & c. $796 \mathrm{C}>\mathrm{T}$ & p.R266* \\
\hline 7 & $\mathrm{~F} / 17 \mathrm{y}$ & $2 \mathrm{~m}$ & c. $796 \mathrm{C}>\mathrm{T}$ & p.R266* & $?$ & $?$ \\
\hline 8 & $\mathrm{~F} / 24 \mathrm{y}$ & $5 \mathrm{~m}$ & c. $796 \mathrm{C}>\mathrm{T}$ & p.R266* & $?$ & $?$ \\
\hline 9 & $\mathrm{~F} / 36 \mathrm{y}$ & $?$ & c. $796 \mathrm{C}>\mathrm{T}$ & p.R266* & c. $796 \mathrm{C}>\mathrm{T}$ & p.R266* \\
\hline 10 & $\mathrm{M} / 22 \mathrm{y}$ & $3 \mathrm{~m}$ & c. $796 \mathrm{C}>\mathrm{T}$ & p.R266* & c.122_127delTGGTCC & p.Leu41fs \\
\hline 11 & $\mathrm{~F} / 51 \mathrm{y}$ & $?$ & c. $796 \mathrm{C}>\mathrm{T}$ & p.R266* & c.522dupT & p.Val175fs \\
\hline 12 & $\mathrm{~F} / 2 \mathrm{y}$ & $2 \mathrm{~m}$ & c. $796 \mathrm{C}>\mathrm{T}$ & p.R266* & c. $455 \mathrm{G}>\mathrm{T}$ & Predicted splicing alteration \\
\hline
\end{tabular}

SERPINB7, serpin peptidase inhibitor, clade B (ovalbumin), member 70; M, male; F, female; w, weeks; m, months; y, years; ?, unknown.

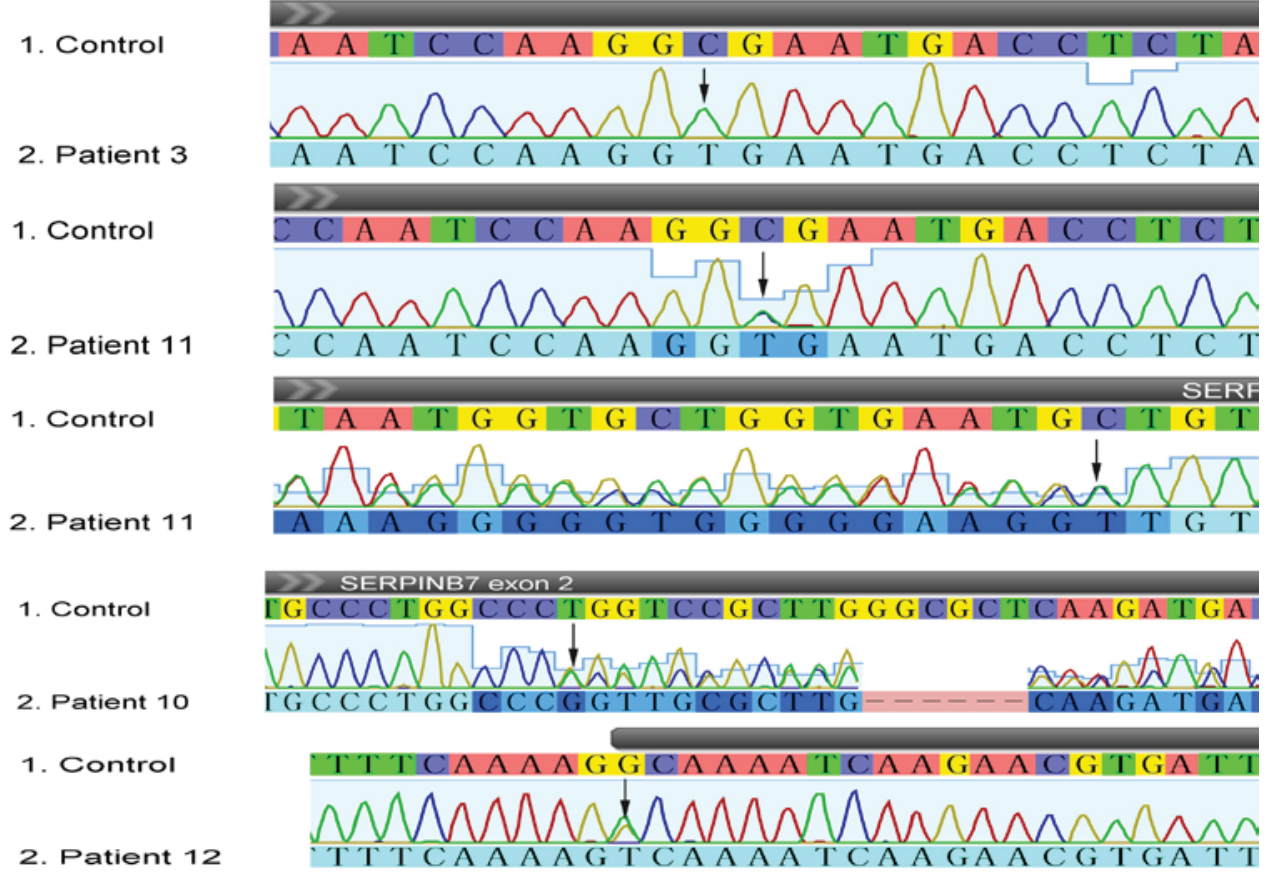

Figure 4. Sequencing results of representative serpin peptidase inhibitor, clade B (ovalbumin), member 70 (SERPINB7) mutations in the present study. From top to bottom, the sequencing results are c.796C $>\mathrm{T}$ (homozygous) in Patient 3, c.796C $>\mathrm{T}$ along with c.522dupT (heterozygous) in Patient 11; c.796C $>\mathrm{T}$ along with c.122_127delTGGTCC (heterozygous) in Patient 10; and c.796C $>\mathrm{T}$ along with c.455G $>\mathrm{T}$ (heterozygous) in Patient 12. Arrows indicate the site of mutation.

reddish appearance in pediatric patients with NPPK (Fig. 2). In addition, other diseases, such as AREI, Unna-Thost type PPK and Bothnia type PPK, are difficult to clinically distinguish, which further underlines the complexity of clinical differential diagnoses between diseases with palmoplantar hyperkeratosis and without other associated features, as well as the importance of molecular analysis.
In conclusion, the present report focused on patients in Mainland China, with respect to nine definite and three suspected patients with NPPK. The results revealed three recurrent and one novel SERPINB7 mutation, thus extending the mutation spectrum of NPPK. Taking the other seven reported Chinese patients, that were definitively diagnosed with NPPK by genetic testing, into account, the present 
Table II. Primers of the six screening genes (SERPINB7, SLURP1, CSTA, AQP5, KRT1 and KRT9).

Primer sequence

\begin{tabular}{|c|c|c|c|}
\hline Primer name & Forward & Reverse & Primer size (bp) \\
\hline SERPINB7-E02 & CAGAAATGTCCACCAACGAG & ATATTTCTGCTGCCTCTTGG & 608 \\
\hline SERPINB7-E03 & CTTTCCTTGTGCCCTGTTTA & TTAAGCTAACCTCCCACCAT & 295 \\
\hline SERPINB7-E04 & GGGCAAGAAAGGATGAAGTT & CATCCCTACCAATAGACACG & 704 \\
\hline SERPINB7-E05 & CCTTCCAGTCCCATTTCCAT & GAGGGTGAGATATTGAGGTT & 615 \\
\hline SERPINB7-E06 & CACAGGGATTATGTAAGGAT & ACACGTTTGGTGGTGTTTCA & 559 \\
\hline SERPINB7-E07 & ACCCAAGGTCACATAGTTAG & CTAGTATCTCAATACCCTGA & 485 \\
\hline SERPINB7-E08 & TCACCTGTCTATTGCTCCAC & ATTGACTTGTGGTGGTTCTT & 765 \\
\hline SLUPR1-E01 & CAGAGGCACAGCCAGGACAT & TAGGAGGTGGGCAGACAAGC & 470 \\
\hline SLUPR1-E02 & TCTGTGGCTCAGCTCAGTTAGA & TCCCTGTTCCCAATAGTCCA & 709 \\
\hline SLUPR1-E03 & TGGACTATTGGGAACAGGGATC & GGTTCAGAGTTCCGAGTTGC & 257 \\
\hline CSTA-E01 & TAAAACACGAGTCTCCACACT & AAAGCCACAAACATCCТАAА & 256 \\
\hline CSTA-E02 & ACTTTTAGGAGGATGAGGTT & AAGGAATTATGTGGTAGGGA & 284 \\
\hline CSTA-E03_NEW & ACCCATTTGAATGAATCTCC & CCAGTTGCATTAGGCTTGAC & 433 \\
\hline AQP5-E01 & CGCCGCATCCACCTCCTCCG & CCCCAGGGTCGAGGCTCCCA & 486 \\
\hline AQP5-E02 & AAAAGCCCTACTCCCCGAGC & GATTCCTGTCCCATCCCACC & 466 \\
\hline AQP5-E03 & CAGGAATCAAACCCAACCTC & TCCCTTTCTCTGTCAGCCAC & 442 \\
\hline AQP5-E04 & CGCTCTGTTCATCCGTCTCT & TTTCTTCTTTTCССССТTGG & 576 \\
\hline KRT1-E01A & CCAAGCCCAАТTTCТTСССТG & AAGGCTCTGGTTGATAGTGA & 549 \\
\hline KRT1-E01B & TGGAAGTCGGAGTCTTGTTA & ATTCAACAGATATGAGTCCC & 696 \\
\hline KRT1-E02 & GTATGCGCTTTGCTATTGGT & ATTGCCTATCACTGCCTTTC & 684 \\
\hline KRT1-E03 & TTAGGTTAGAGGCACATCAG & AAATGTGAGTTCCGTCCTAC & 313 \\
\hline KRT1-E04+05 & CCATATTTCCCAGCACCTTA & AGATGGTAGATAGCGTTTGT & 794 \\
\hline KRT1-E06 & CAAGGTGAGTGGGCTGAAAG & CTCACATTGACCATCCCATC & 492 \\
\hline KRT1-E07 & AGTCTGTAAGGGTTGTAGGAG & GAATAATTTGCTCCACCTCA & 699 \\
\hline KRT1-E08+09 & GCGGTTTGGGAAGCTGGAGT & TTGAAATGTCATGTGGGTGG & 877 \\
\hline KRT9-E01_in & CGGTAGCACTCCTATCACTGC & CTGCTCTGCCCAAACTCTGAA & 931 \\
\hline KRT9-E02+03 & ATCTTCGCTGAAGGCTGGAA & AAGCCAAAGCCCAACCACTA & 701 \\
\hline KRT9-E04 & GTGGTTGGGCTTTGGCTTCA & GGAGGTGGGAGGGATGGAGA & 356 \\
\hline KRT9-E05+06 & GACTTGTCATTGGCTTCAGA & CAGAGGGACAGAAGTAGTATCA & 664 \\
\hline KRT9-E07 & AGATTCATGTTTGGGTCCTG & CCCTTACCTTTTGTCTCATCT & 621 \\
\hline
\end{tabular}

Table III. Allele frequencies of known pathogenic mutations (in bold) and other potential pathogenic variants in distinct population.

\begin{tabular}{|c|c|c|c|c|c|c|c|c|}
\hline \multirow[b]{2}{*}{ Mutation } & \multirow{2}{*}{$\begin{array}{c}\text { Nucleotide } \\
\text { change }\end{array}$} & \multirow{2}{*}{$\begin{array}{l}\text { Amino acid } \\
\text { change }\end{array}$} & \multicolumn{6}{|c|}{ Allele frequencies in distinct populations } \\
\hline & & & Han & Japanese & African & American & European & All \\
\hline rs201433665 & c. $336+2 \mathrm{~T}>\mathrm{G}$ & p.? & 0.0025 & 0 & 0 & 0 & 0 & $<0.001$ \\
\hline rs202182550 & $c .455 G>T$ & p.? & 0.0025 & 0 & 0 & 0 & 0 & $<0.001$ \\
\hline rs142859678 & c.796C $>\mathrm{T}$ & p.Arg $266^{*}$ & 0.017 & 0.014 & 0 & 0 & 0 & 0.002 \\
\hline rs182539714 & c. $-16 \mathrm{~T}>\mathrm{A}$ & p.? & 0.005 & 0.005 & 0 & 0 & 0 & 0.001 \\
\hline rs199666937 & c. $140 \mathrm{~A}>\mathrm{G}$ & p.Gln47Arg & 0 & 0 & 0 & 0 & 0.001 & $<0.001$ \\
\hline rs74653657 & c. $181 \mathrm{~A}>\mathrm{G}$ & p.Asn61Asp & 0.012 & 0 & 0 & 0 & 0 & 0.002 \\
\hline rs201239910 & c. $388 \mathrm{G}>\mathrm{A}$ & p.Asp130Asn & 0 & 0 & 0.001 & 0 & 0 & $<0.001$ \\
\hline rs201821537 & c. $219+8 C>A$ & p.? & 0 & 0 & 0 & 0.001 & 0 & $<0.001$ \\
\hline rs186928560 & c. $220-6 \mathrm{~T}>\mathrm{G}$ & p.? & 0 & 0 & 0 & 0 & 0.001 & $<0.001$ \\
\hline rs139542928 & c. $943 \mathrm{C}>\mathrm{T}$ & p.Arg 315Cys & 0 & 0 & 0.001 & 0 & 0 & $<0.001$ \\
\hline rs201208667 & c. $1136 \mathrm{G}>\mathrm{A}$ & p.Cys379Tyr & 0 & 0 & 0 & 0 & 0.001 & $<0.001$ \\
\hline
\end{tabular}

?, unknown. 
study further demonstrated that NPPK is a common entity in Mainland China, and c.796C $>\mathrm{T}$ is the most prevalent mutation and exerts a founder effect. At present, there is no effective drug treatment for the majority of diffuse and severe forms of PPK, whereas the symptoms of NPPK are relatively milder and long-time prognosis is favorable. Considering the high allele frequency of the founder mutation, genetic counseling is essential for patients with NPPK or carriers of NPPK.

\section{Acknowledgements}

The present study was supported by grants from the Industry Foundation of Ministry of Health of China (grant no. 20120213), the Ph.D. Programs Foundation of Ministry of Education of China (grant no. 20130073120014), the Natural Science Foundation of Shanghai Jiaotong University School of Medicine (grant no. 13XJ10023) and a grant from the Foundation of Xinhua Hospital affiliated to Shanghai Jiaotong University School of Medicine (grant no. 15YJ15).

\section{References}

1. Kabashima K, Sakabe J, Yamada Y and Tokura Y: 'Nagashima-type' keratosis as a novel entity in the palmoplantar keratoderma category. Arch Dermatol 144: 375-379, 2008.

2. Yin J, Xu G, Wang H, Zhao J, Duo L, Cao X, Tang Z, Lin Z and Yang Y: New and recurrent SERPINB7 mutations in seven Chinese patients with Nagashima-type palmoplantar keratosis. J Invest Dermatol 134: 2269-2272, 2014.

3. Kubo A, Shiohama A, Sasaki T, Nakabayashi K, Kawasaki H, Atsugi T, Sato S, Shimizu A, Mikami S, Tanizaki H, et al: Mutations in SERPINB7, encoding a member of the serine protease inhibitor superfamily, cause Nagashima-type palmoplantar keratosis. Am J Hum Genet 93: 945-956, 2013.

4. Moosbrugger-Martinz V, Jalili A, Schossig AS, Jahn-Bassler K, Zschocke J, Schmuth M, Stingl G, Eckl KM, Hennies HC and Gruber R: Epidermal barrier abnormalities in exfoliative ichthyosis with a novel homozygous loss-of-function mutation in CSTA. Brit J Dermatol 172: 1628-1632, 2015.

5. Gruber R, Hennies HC, Romani N and Schmuth M: A novel homozygous missense mutation in SLURP1 causing Mal de Meleda with an atypical phenotype. Arch Dermatol 147: 748-750, 2011.
6. Mizuno O, Nomura T, Suzuki S, Takeda M, Ohguchi Y, Fujita Y, Nishie W, Sugiura K, Akiyama M and Shimizu H: Highly prevalent SERPINB7 founder mutation causes pseudodominant inheritance pattern in Nagashima-type palmoplantar keratosis. Br J Dermatol 171: 847-853, 2014.

7. Hida T, Okura M, Kamiya T and Yamashita T: Nagashima-type palmoplantar keratosis caused by compound heterozygous mutations in SERPINB7. Eur J Dermatol 25: 202-203, 2015.

8. Mokni M, Charfeddine C, Ben Mously R, Baccouche D, Kaabi B, Ben Osman A, Dellagi K and Abdelhak S: Heterozygous manifestations in female carriers of Mal de Meleda. Clin Genet 65: 244-246, 2004.

9. EcklKM,StevensHP,LestringantGG,Westenberger-TreumannM, Traupe H, Hinz B, Frossard PM, Stadler R, Leigh IM, Nürnberg P, et al: Mal de Meleda (MDM) caused by mutations in the gene for SLURP-1 in patients from Germany, Turkey, Palestine, and the United Arab Emirates. Hum Genet 112: 50-56, 2003.

10. Bchetnia M, Laroussi N, Youssef M, Charfeddine C, Ben Brick AS, Boubaker MS, Mokni M, Abdelhak S, Zili J and Benmously R: Particular Mal de Meleda phenotypes in Tunisia and mutations founder effect in the Mediterranean region. Biomed Res Int 2013: 206803, 2013.

11. Kubo A: Nagashima-type palmoplantar keratosis: A common Asian type caused by SERPINB7 protease inhibitor deficiency. J Invest Dermatol 134: 2076-2079, 2014.

12. Silverman GA, Whisstock JC, Askew DJ, Pak SC, Luke CJ, Cataltepe S, Irving JA and Bird PI: Human clade B serpins (ov-serpins) belong to a cohort of evolutionarily dispersed intracellular proteinase inhibitor clades that protect cells from promiscuous proteolysis. Cell Mol Life Sci 61: 301-325, 2004.

13. Sakabe JI, Kabashima K, Sugita K and Tokura Y: Possible involvement of $\mathrm{T}$ lymphocytes in the pathogenesis of Nagashima-type keratosis palmoplantaris. Clin Exp Dermatol 34: e282-e284, 2009.

14. Sartore L, Bordignon M, Bassetto F, Voltan A, Tomat V and Alaibac M: Melanoma in skin affected with keratoderma palmoplantaris hereditaria (Mal de Meleda): Treatment with excision and grafting. J Am Acad Dermatol 61: 161-163, 2009.

15. He Y, Zeng K, Zhang X, Chen Q, Wu J, Li H, Zhou Y, Glusman G, Roach J, Etheridge A, et al: A gain-of-function mutation in TRPV3 causes focal palmoplantar keratoderma in a Chinese family. J Invest Dermatol 135: 907-909, 2014.

16. Zhang J, Cheng R, Ni C, Liang J, Li M and Yao Z: First Mal de Meleda report in Chinese Mainland: Two families with a recurrent homozygous missense mutation in SLURP-1. J Eur Acad Dermatol Venereol 30: 871-873, 2016.

17. Nakamizo S, Katoh N, Miyachi Y and Kabashima K: Atypical nail dystrophy in a possible case of Nagashima-type palmoplantar keratosis. J Dermatol 39: 470-471, 2012. 